\title{
Wohlfahrt neu gemischt
}

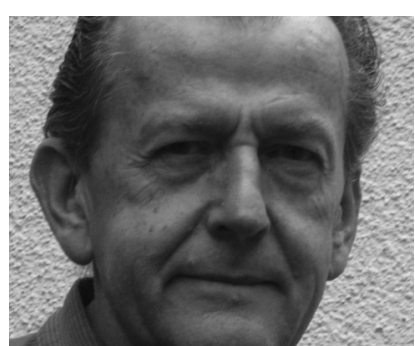

VON WOLF RAINER WENDT Prof. Dr. Wolf Rainer Wendt ist Honorarprofessor der Eberhard Karls Universität Tübingen. Er ist Vorsitzender der Deutschen Gesellschaft für Care und Case Management. Seit langem ist er Mitglied im Beirat der Zeitschrift SOZIALwirtschaft und des Informationsdienstes SOZIALwirtschaft aktuell sowie Herausgeber der Buchreihe "Forschung und Entwicklung in der Sozialwirtschaft« in der Nomos Verlagsgesellschaft, Baden-Baden.

E-Mail wendt@imails.de
Angesichts der Pluralisierung von Lebenslagen und der Emanzipation der Nutzer lassen sich soziale Dienstleistungen immer weniger fertig bereitstellen. Vielmehr müssen in jedem Einzelfall personen- und situationsbezogene Hilfe-Pakte in Partnerschaft mit den Klienten und seinem Umfeld ausgehandelt werden. Personalisierte Versorgungsgestaltung wird zu einem Megatrend für die Sozialwirtschaft.

Es liegt im Trend zur Kooperation und Integration in Humandiensten, nicht nur verschiedene professionelle Praktiken fachgebiets- und sektorübergreifend zu verbinden, sondern in der sozialen Sorge und Versorgung auch informelle Hilfen heranzuziehen und auf die Eigenleistungen der Adressaten zu bauen.

Damit ändern sich die » Produktionsweise « von Wohlfahrt und die darauf gerichtete Gestaltung von Versorgung. Das Leistungsgeschehen verlagert sich tendenziell aus dem institutionellen Raum der Daseinsvorsorge in den Lebens- und Beziehungsraum selbstaktiver oder $\mathrm{zu}$ aktivierender Akteure.

In Verbindung mit ihnen wird Partnerschaft in der sozialen Leistungserbringung gesucht, und sie bringen in diese Partnerschaft ihre Art und Weise des Zurechtkommens ein. Mit neuer Teilung von Verantwortung für Versorgung wandeln sich auf der Individualebene die Positionen im sozialrechtlichen Dreiecksverhältnis. Personalisierte Versorgungsgestaltung wird zu einer eigenen sozialwirtschaftlichen Aufgabe.

\section{Verlagerung der Leistungserstellung}

Schon seit längerer Zeit ist eine Standortverschiebung von Einrichtungen und Diensten hin zu den Nutzern zu beobachten. Was mit der Deinstitutionalisierung dem Abbau stationärer Strukturen und der Vermeidung von Dauerunterbringungen begonnen wurde und mit dem Vorrang ambulanter Hilfen fortgesetzt wurde, kommt bei häuslicher Unterstützung an. In der Jugendhilfe und in der Behindertenhilfe werden Heimunterbringungen fernab von $\mathrm{Fa}-$ milie und Wohnort möglichst vermieden und Betreuungsangebote im Lebensfeld bevorzugt. In der Altenhilfe sind Hilfen im Quartier gefragt, damit ein Pflegebedürftiger im eigenen Haushalt bleiben kann. Dem Zug in den Nahraum muss der Zuschnitt der Dienstleistungen folgen; er hat sich lokalen und individuellen Anforderungen anzupassen. Die herkömmliche Angebotsorientierung bietet diesen Zuschnitt nicht.

»Insassen « einer Einrichtung können sich dem »Angebot « dort nicht entziehen. Im individuellen Lebensfeld aber muss sich ein Dienstleister mit den Besonderheiten der Alltagsgestaltung und auch dem Eigensinn der Klienten arrangieren. Wenn aus den professionellen Bemühungen nichts wird, kann das auch daran liegen, dass der Adressat sich selber ohne oder mit den gebotenen Hilfen anders zu helfen weiß oder geholfen hat, als es den professionellen Intentionen entspricht.

Im Wohlfahrtsmix steuert jeder Akteur auf seine Weise. Dazu müssen die formell und informell Beteiligten sich aufeinander beziehen können. »Nähe « und »Teilhabe « bedingen sich. Zum Beispiel wird von einem Pflegestützpunkt das örtliche Versorgungsangebot dort in Vernetzung erschlossen, wo es die Nutzer brauchen können und zwar so, dass diese sich in ihrem Quartier nicht aus ihrem informellen Netzwerk sozialer Beziehungen und Unterstützungsmöglichkeiten entfernen müssen.

Einrichtungen und Dienste werden, damit sie hier und da und ganz verschieden genutzt werden können, auf einer Plattform präsentiert und gehen in ein Netzwerk im Quartier über. Niedrigschwellig gehören sie zur Nachbarschaft, ergänzen oder kompensieren sie. Die Verbindung zu den Nutzern wird informationstechnologisch unterstützt - man spricht von einem »virtuellen " oder »digitalen Stadtquartier " (Bundesministerium 2007, 30). Einer Versorgung im Verbund von formellen Angeboten und informellen Hilfen dienen auch die Mehrgenerationenhäuser, Familienzentren und Familienkompetenzzentren als Knoten in Netzwerken von Dienstleistern und von Nutzern. Mit eingeschalteten Servicestellen wird dabei den veränderten $\mathrm{Ak}$ teurkonstellationen Rechnung getragen (Schneiders 2010). 
Die Nutzer personenbezogener Dienste wirken bei deren Ausformung mit. Sie haben über ihr Wunsch- und Wahlrecht hinaus ihre Muster, was wie getan werden und - nicht zuletzt mit eigenen Kräften - geleistet werden kann. »Personalisierung durch Teilhabe ", hieß die Losung, die 2004 für soziale Dienstleistungen in Großbritannien ausgegeben wurde (Leadbetter 2004). Gefragt ist insbesondere eine individualisierte Zusammenstellung von Hilfen, dem Bedarf entsprechend nebeneinander und nacheinander. Sie gehen in ein Versorgungskontinuum ein, das bei Gefährdungen des Kindeswohls, in Multiproblemfamilien, bei Behinderung, bei chronischen gesundheitlichen Belastungen oder Pflegebedürftigkeit zur andauernden Lebensbewältigung gehört. aber auch auf spezialisierte Kompetenzund hochqualifizierte Versorgungszentren stützen können, welche die Leistungen bieten, deren dezentrale Bereitstellung nicht wirtschaftlich wäre.

Musterhaft geregelt findet sich die Organisation betrieblicher und personenbezogener Kooperation unter kommunaler Regie im seit 2007 in den Niederlanden geltenden "Gesetz gesellschaftlicher Unterstützung " (Wet maatschappelijk ondersteuning, Wmo). Es richtet sich auf förderliche Bedingungen, unter denen Menschen bei Pflegebedürftigkeit oder Behinderung von Angehörigen und freiwilligen Helfern versorgt werden, wozu die Selbständigkeit der Bürgerinnen und Bürger gestärkt und ihre aktive Beteiligung unter kommunaler Regie eingefordert wird. Die lokale soziale

und passende Unterstützungsstrukturen erhalten, abwandeln und entwickeln. Hilfreich sind dabei Plattformen, auf denen dauernd ein Austausch der informell und formell Beteiligten erfolgen kann, durch den ein Vorrat an Kompetenz gebildet wird, von dem sich in vielen Fällen zehren lässt.

Indem sie solche Plattformen bereitstellen, können professionelle Service- und Kompetenzagenturen eine Scharnierfunktion übernehmen. Sie übernehmen einen Auftrag im lokalen Care Management entweder für eine bestimmte Zielgruppe (z. B. in der Migrationsberatung oder in der Beschäftigungsförderung Jugendlicher) oder für bereichsspezifische Koordinationsaufgaben (etwa in der Familienhilfe). Eine besondere Rolle können Selbsthilfezusammenschlüsse als Partner in der gesundheitlichen Versorgungsgestaltung spielen (Trojan 2010).

\section{Zur Lebensbewältigung beitragen}

\section{"Im Wohlfahrtsmix steuert jeder Akteur auf seine Weise"}

Die Verbindung von Hilfemöglichkeiten kann nicht nur durch ihre externe Einbeziehung erfolgen, sondern auch in einer nutzergebundenen internen Versorgung. Es ist der Sinn von Sozial- und Assistenzgenossenschaften, dass Leistungen nicht von außen geliefert und von Leistungsberechtigten nur abgeholt werden müssen, sondern dass die Gestaltung des ganzen Prozesses eine gemeinsame Angelegenheit der in Selbstsorge und von in Fürsorge Beteiligten ist. Sie sind ermächtigt zum Improvisieren und zu eigenständiger Vernetzung informellen Engagements und formeller Unterstützung.

\section{Passende Arrangements der Versorgung gestalten}

Wirksame Beteiligung wird schon deshalb benötigt, weil der Sozialstaat überfordert ist, sein System der Sozialleistungen in deren detaillierter Erbringung angemessen zu steuern. Er hat keinen Durchgriff auf das diskrete Leistungsgeschehen und ist darauf angewiesen, dass in Abstimmung und $\mathrm{Zu}-$ sammenarbeit der Akteure auf unteren Ebenen und nah an den Problemen mittelbar dasjenige "Regieren « des Systems erfolgt, das der Staat selber nicht fertigbringt. Gefragt ist eine partnerschaftliche Praxis des Regierens (Governance) im Feld der Daseinsvorsorge und der sozialen und gesundheitlichen Versorgung in ihren einzelnen Bereichen. Sie will lokal und nachbarschaftlich angelegt sein, vielerlei Befähigungen und freies Engagement einschließen, sich
Infrastruktur soll so eingerichtet sein, dass alle teilhaben (können). Man spricht von einem »Partizipationsgesetz «; es verbindet eine vertikale Verantwortung der öffentlichen Hand mit der horizontalen Verantwortung mit Miteinander partnerschaftlicher Sorge (vgl. Klerk/Gilsing/Timmermans 2010).

Herkömmlich finden wir die öffentliche Daseinsvorsorge, die Versorgungsgestaltung und die betriebliche Steuerung von Versorgung abgehoben von der Lenkung des personenbezogenen Vorgehens, der individuellen Fallführung. Dispositionen auf der Organisationsebene haben wenig mit der Entscheidungsfindung im Einzelfall zu tun. Organisation und Methode schneiden sich nicht. Erst die Integration von Sorge und Versorgung bringt mit sich, dass die Methode die Organisation braucht und der ganze Betrieb der Versorgung zu seiner Individualisierung einer Entsprechung in der managerialen Vorgehensweise im Einzelfall bedarf. Das personenzentrierte Prozessmanagement verbindet sich in dem Umfeld, in dem es nötig wird, mit einem Netzwerkmanagement in Zusammenführung und Entwicklung geeigneter Versorgungsmöglichkeiten - beispielsweise im Stadtquartier (Bundesministerium 2007).

Die individuellen Arrangements kommen per » merging and matching " zustande. Das heißt, dienstliche Versorgung schließt sich mit dem Sorgehandeln von Personen zusammen, indem sie in ihren Vorhaben und ihren Möglichkeiten übereinkommen
Der Trend zur Integration hebt im Versorgungssystem herkömmliche Abgrenzungen von Fachgebieten und Zuständigkeiten im Sozial- und Gesundheitswesen auf. Professionalität in Humandiensten muss sich neu positionieren. Erwartet wird ein breites Sichkümmern um eine in der Regel komplexe Problematik. Ihre sozialwirtschaftliche Bewältigung erfolgt in einer Wechselbeziehung

- von Organisations- und Steuerungserfordernissen im persönlichen Leben und Lebensumfeld einerseits und

- Organisations- und Steuerungserfordernissen in der humandienstlichen Infrastruktur andererseits.

Menschen sind in ihrer Lebensgestaltung gefordert: Familiengründung und Kindererziehung, soziale Beziehungspflege, berufliche Qualifizierung, Haushaltsführung, Balance von Elternschaft und Erwerbsarbeit, Bewältigung von Behinderung oder Krankheit. Die Gestaltung von dienstlicher Versorgung richtet sich darauf komplementär und kompensatorisch ein. Die Anforderungen wandeln sich und variieren lokal je nach Bevölkerungsstruktur, Lage auf dem Arbeitsmarkt, Wohnverhältnissen usw. Ein Care Management hat die Wechselbeziehung von Lebensgestaltung und Versorgungsgestaltung überindividuell (im Sozialraum und im Wohlfahrtsmix) und personalisiert im Blick.

Exemplarisch für diese Entwicklung ist im Rahmen von Care die Ausdehnung von Case Management. Nach ihm wird überall verlangt, wo man kontinuierlich und vielseitig Dienste verknüpfen und Zusammenarbeit herbeiführen muss. Ein Case Management nimmt in Pflegestützpunkten 
gleichzeitig Erfordernisse sozialer, pflegepraktischer, medizinischer, psychologischer und hauswirtschaftlicher Art in den Blick und knüpft an sie diverse Hilfemöglichkeiten und Dienste:

- In der Eingliederung behinderter Menschen kann es nicht bei einem bloßen Nach- und Nebeneinander medizinischer, beruflicher und sozialer Rehabilitationsmaßnahmen bleiben. Die Gestaltung eines Lebens mit Behinderung ist zu begleiten.

- Das beschäftigungsorientierte Fallmanagement in den Rechtskreisen des SGB II und SGB III zieht um den Angelpunkt der Arbeitssuche Kreise in persönlichen Lebensbereichen, arbeitet an den in ihnen gegebenen Vermittlungshemmnissen und baut auf vorhandene Kompetenzen.

- Jugendhilfe, die sich gleichzeitig um Familienverhältnisse und um sozialräumliche Bedingungen des Jugendlebens kümmert, bietet Lösungen à la carte, sondern erschließt sie per Hilfeplanung und bewegliche Ausführung in einem örtlichen Kooperationsnetzwerk.

\section{Über Sorgeberatung zur Versorgungsgestaltung}

Sozialwirtschaftliche Leistungen lassen sich weniger fertig bereitstellen, als dass sie in Partnerschaft mit ihren Nutzern nach Aushandlung personen- und situationsbezogen generiert werden. Was sich machen lässt, ergibt sich in einem Beratungsprozess. Da die Problemlagen meist komplex sind, muss umsichtig nach Rat gesucht werden, wobei die konkreten Lebensverhältnisse und Handlungsmöglichkeiten im Einzelfall wie die Gegebenheiten des Versorgungssystems in Betracht zu ziehen sind. Die Konsultation, die nach dem Konzept der Sorgeberatung breit angelegt ist und nicht wie im herkömmlichen Beratungsangebot spezialisiert erfolgt, zielt auf eine angemessene Gestaltung nötiger Versorgung.

Beraten wird darüber, wie jemand in einer Problemlage mit seinen Möglichkeiten und unter Beiziehung verschiedener Hilfen sein Leben oder das seiner Familie gestalten kann - oder wie in derartigen Situationen per Arrangement von Diensten eine geeignete Versorgungsgestaltung erreicht wird. Im ersten Fall ist Rat zur selbständigen Lebensführung (z. B. von Alleinerziehenden mit mehreren Kindern, bei chronischer Erkrankung, Behinderung oder Pflegebedürftigkeit) und der dazu nötigen Ausstattung gesucht. Im anderen Fall besteht die Hauptaufgabe in der Koordination des Dienstleistungsgeschehens. Rat ist in ihm - in seiner personalisierten Anpassung, Um- und Neugestaltung - zu suchen.
Mancherorts denkt man an die Ausprägung eines eigenen Berufsbildes Versorgungsgestaltung, zuständig z. B. für integrierte Versorgung bei Demenz (SchäferWalkmann/Deterding 2009). Professionell verbindet sich hier ein Case Management in der Einzelhilfe mit einem Care Management in der lokalen oder regionalen Steuerung des Systems sozialer und gesundheitlicher Dienstleistungen inklusive informeller und bürgerschaftlicher Unterstützung. Die sozialwirtschaftliche Regie verklammert bedarfsorientiert individuelles Bewältigungshandeln mit der Organisation der Dienste.

Literatur:

Bundesministerium für Verkehr, Bau und Stadtentwicklung: Stadtquartiere für Jung und Alt. Das ExWoSt-Forschungsfeld "Innovationen für familien- und altengerechte Stadtquartiere». Berlin, September 2007.

Bartnik, Eddi/Chalmers, Ron: It's about More than the Money. Local Area Coordination Supporting People with Disabilities. In: Hunter, Susan/Ritchie, Pete (eds.): Co-Production and Personalisation in Social Care. Changing Relationships in the Provision of Social Care. Jessica Kingsley, London 2008. S. 19-38. Hunter, Susan/Ritchie, Pete (eds.): Co-Production and Personalisation in Social Care. Changing Relationships in the Provision of Social Care. Jessica Kingsley, London 2008.

Klerk, Mirjam de/Gilsing, Rob/Timmermans, Joost (red.): Op weg met de Wmo. Evaluatie van de Wet maatschappelijke ondersteuning 20072009. Sociaal en Cultureel Planbureau, Den Haag, mart 2010.

Leadbetter, Charles: Personalisation through Participation. A New Script for Public Services. Demos, London 2004.

Schäfer-Walkmann, Susanne/Deterding, Dorothee: Demenzversorgung integriert gestalten. Impulse aus dem "Leuchtturmprojekt Demenz". In: Forum sozialarbeit + gesundheit 4/2009, S. 25-28.

Schneiders, Katrin: Vom Altenheim zum Seniorenservice. Institutioneller Wandel und Akteurkonstellationen im sozialen Dienstleistungssektor. Nomos, Baden-Baden 2010. Trojan, Alf: Selbsthilfegruppen in der Versorgungsgestaltung: Von der Kooperation zu nachhaltiger Beteiligung. In: Dahme, Heinz-Jürgen/Wohlfahrt, Norbert (Hg.): Systemanalyse als politische Reformstrategie. VS Verlag für Sozialwissenschaften, Wiesbaden 2010. S. 347369.

\section{Der „Klassiker“}

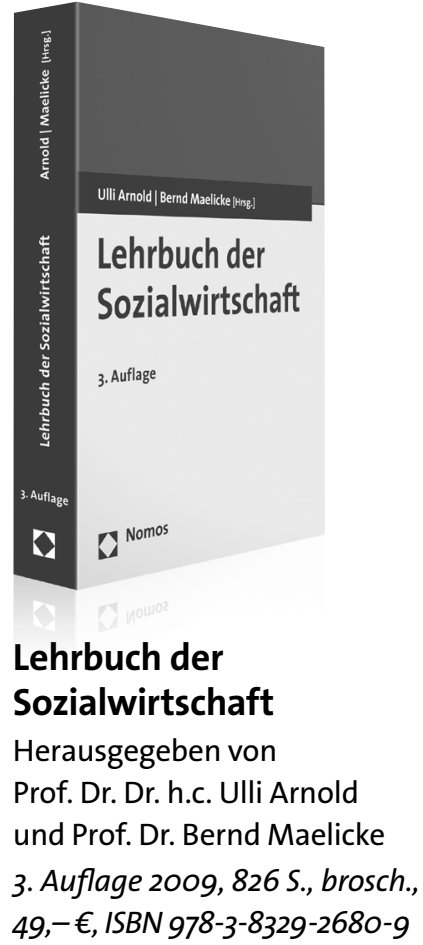

Die rapide Veränderung der politischen und strukturellen Rahmenbedingungen für die Sozialwirtschaft erfordert grundlegende Änderungen in der Ausbildung und Weiterbildung von Führungskräften sozialer Dienstleistungsorganisationen.

Die vollständig aktualisierte und neu bearbeitete 3. Auflage vermittelt Basiswissen und aktuelle Informationen zu den wichtigsten Themen wie z.B.:

- Entwicklung der sozialpolitischen Voraussetzungen

- Anbieter, Wettbewerber, Handlungsfelder, Dienstleistungen

- Typologie und Rechtsformen sozialwirtschaftlicher Organisationen

- Qualitätsmanagement, Sozialmarketing

- Finanzierungsmanagement, Fundraising

- Grundlagen des Managements in der Sozialwirtschaft

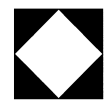

